\title{
Apparent diffusion coefficient values detected by diffusion-weighted imaging in the prognosis of patients with locally advanced esophageal
} squamous cell carcinoma receiving chemoradiation

\author{
This article was published in the following Dove Press journal: \\ OncoTargets and Therapy \\ 21 September 2016 \\ Number of times this article has been viewed
}

\begin{abstract}
Shu Liu',*
Fuxi Zhen ${ }^{2, *}$

Nana Sun ${ }^{3}$

Jiayan Chen'

Yuandong $\mathrm{CaO}^{\prime}$

Sheng Zhang'

Hongyan Cheng ${ }^{4}$

Xiaolin $\mathrm{Ge}^{1}$

Xinchen Sun'

'Department of Radiation Oncology, the First Affiliated Hospital of

Nanjing Medical University, Nanjing,

Jiangsu, People's Republic of China;

${ }^{2}$ Department of Thoracic Surgery,

the First Affiliated Hospital of

Nanjing Medical University, Nanjing, Jiangsu, People's Republic of China;

${ }^{3}$ Department of Radiology, the First Affiliated Hospital of Nanjing Medical University, Nanjing, Jiangsu, People's Republic of China; ${ }^{4}$ Department of General Internal Medicine, the First Affiliated Hospital of Nanjing Medical University, Nanjing, Jiangsu, People's Republic of China

*These authors contributed equally to this work
\end{abstract}

Correspondence: Xiaolin Ge;

Xinchen Sun

Department of Radiation Oncology, the First Affiliated Hospital of Nanjing Medical University, Jiangsu Province Hospital, 300 Guanzhou Road, Nanjing 210029, People's Republic of China $\mathrm{Tel} / \mathrm{fax}+862568135700$

Email doctorgx|@163.com; sunxinchen20I2@I63.com
Purpose: Previous studies have demonstrated that apparent diffusion coefficient (ADC) values measured by magnetic resonance imaging have prognostic value in patients with esophageal squamous cell carcinoma (ESCC). However, the role of ADC needs to be validated in a cohort of Chinese ESCC patients. This study assessed the role of ADC in predicting the outcome of patients with ESCC treated only by chemoradiation in the People's Republic of China.

Patients and methods: Seventy-three patients with local advanced ESCC were retrospectively analyzed in this study; none of the patients underwent surgery before or after chemoradiation. The ADC values of the primary tumors were determined by magnetic resonance imaging. The ADC values were then correlated with clinicopathological and other radiological parameters. Survival analysis was carried out to determine if ADC had an impact on survival of these patients.

Results: The median ADC value of the esophageal cancer tissue was $1.256^{*} 10^{-3} \mathrm{~mm}^{2} / \mathrm{sec}$ (range: $0.657-2.354 * 10^{-3} \mathrm{~mm}^{2} / \mathrm{sec}$, interquartile range $0.606 * 10^{-3} \mathrm{~mm}^{2} / \mathrm{sec}$ ). No clinicopathological or radiological parameters were associated with the ADC values except the sites of tumor tissues. ADC $<1.076^{*} 10^{-3} \mathrm{~mm}^{2} / \mathrm{sec}$ predicted significantly worse survival in patients with ESCC (12.9 months vs undefined, $P=0.0108$ ).

Conclusion: The ADC value is a potent prognostic factor which can be used to predict the outcome of patients with ESCC treated only by chemoradiation.

Keywords: esophageal cancer, radiation, prognosis, ADC, chemotherapy, diffusionweighted MRI

\section{Introduction}

Esophageal cancer remains a worldwide health problem, as it is the fourth most common cause of cancer deaths. ${ }^{1}$ Surgery constitutes the backbone of treatment for resectable disease; however, for patients with unresectable disease or contraindication to esophagectomy, chemoradiation therapy (CRT) is the mainstay of treatment. ${ }^{1}$ Although improved prognosis has been achieved in patients treated with CRT, side effects caused by chemoradiation including bone marrow suppression, esophagitis, pericarditis, and pneumonia should also be considered in the clinical setting. ${ }^{2}$ As a result, it is increasingly important to predict the response to CRT in order to identify patients who can benefit best from CRT while avoiding unnecessary adverse events.

Published studies have demonstrated that clinical and histopathological factors, as well as biomarkers and functional imaging could predict the response to CRT. 
Functional magnetic resonance imaging (MRI) can be used to detect alterations in tumor pathophysiology. Different tissues have unique diffusion characteristics, as revealed by the apparent diffusion coefficient (ADC), which can be determined from the diffusion-weighted imaging (DWI) measurements. Several studies have investigated the role of ADC in predicting the response to CRT and survival in patients with esophageal cancer. ${ }^{2,3}$ However, the validation of the prognostic role of ADC in a cohort in Chinese patients is lacking. Therefore, we retrospectively analyzed the efficacy of ADC in predicting the response to CRT and survival in patients in our institute.

\section{Materials and methods}

\section{Patients}

Seventy-three patients (54 men, 19 women; median age 63, range [46-85], interquartile range [12.5]) with esophageal squamous cell carcinoma (ESCC) admitted to our center from January 2011 to 2015 were included into this study. All patients had an upper endoscopic examination with tumor biopsy, barium esophagography, and chest and abdominal computed tomography (CT) scans to determine their clinical stages. In seventy-three patients, eight patients were treated with radiotherapy alone, and the remaining patients were treated with chemoradiation. None of the patients underwent surgery before or after chemoradiation. There was one patient in stage 1, four in stage 2, 30 in stage 3 , and 38 in stage 4 . Written informed consent was obtained from all the patients according to the Declaration of Helsinki and the study was approved by the review boards of the first affiliated hospital of Nanjing Medical University.

\section{Chemoradiation}

Gross tumor volume (GTV) was delineated by using available resources, including diagnostic CT, barium esophagography, and endoscopic reports. It was defined as visible primary tumor and involved lymph nodes (minor diameter $>1 \mathrm{~cm}$ on CT scan). Clinical target volume was defined as GTV of primary tumor plus a $3 \mathrm{~cm}$ margin craniocaudally with $0.5-0.8 \mathrm{~cm}$ lateral margins and GTV of involved nodes. Planning target volume (PTV) was GTV plus a uniform $0.3 \mathrm{~cm}$ expanded margin. The prescription dose to cover $95 \%$ of PTV was $50 \mathrm{~Gy}$. The dose delivered to the primary tumor site and involved lymph node was increased to $60 \mathrm{~Gy}$ (2Gy/ fraction, five fractions/week). Concurrent chemotherapy was used as follows: liposomal paclitaxel $35 \mathrm{mg} / \mathrm{m}^{2}$ plus cisplatin $25 \mathrm{mg} / \mathrm{m}^{2}$ was administered on day 1 weekly, for 6 weeks.

\section{MRI protocols}

Examination was performed with a 3.0-T MR scanner (Verio Tim; Siemens, Erlangen, Germany) and a 16-channel torso coil. The sequences included: axial T1-weighted volumetric interpolated breath-hold examination fat-suppressed images (repetition time msec/echo time msec, 4.56/2.03; slice thickness, $5 \mathrm{~mm}$; field of view, $38 \times 30 \mathrm{~cm}$; matrix, $320 \times 182$ ); axial T2-weighted turbo spin-echo images (repetition time msec/echo time msec, 3,550/86; slice thickness, $5 \mathrm{~mm}$; field of view, $22 \times 22 \mathrm{~cm}$; and matrix, 384×269); sagittal breath-hold T2-weighted half-Fourier acquisition single-shot turbo spin-echo images (repetition time msec/effective echo time msec, 1,200/93; slice thickness, $5 \mathrm{~mm}$; field of view, $35 \times 35 \mathrm{~cm}$; and matrix, $384 \times 269$ ); parameters of DWI were as follows: repetition time msec/effective echo time msec, 6,400/82; slice thickness, $5 \mathrm{~mm}$; field of view, $38 \times 28 \mathrm{~cm}$; matrix, $128 \times 96$; b-values of $0,800 \mathrm{sec} / \mathrm{mm}^{2}$.

\section{Region of interest placement}

Measurements were obtained on the ADC maps that were reconstructed by using the $b$-value of 0 and $800 \mathrm{sec} / \mathrm{mm}^{2}$. The tumor tissue is characterized by high-signal intensity on DWI and low-signal intensity on ADC maps (Figure 1). On the ADC images, three region of interests (ROIs) (area range, 22.42-41.21 $\mathrm{mm}^{2}$; mean, $32.41 \mathrm{~mm}^{2}$ ) were manually drawn on the solid sections of the tumors. Referring to T2-weighted image, visually identified vessels and necrotic areas were avoided. The measured ADC values from three ROIs were then averaged to a mean value for future statistical analysis.

\section{Statistics}

The Student's $t$-test or Mann-Whitney $U$-test was used for comparison of continuous variables between subgroups. D'Agostino-Pearson omnibus normality test was used to test if the values came from Gaussian distribution. If the values passed normality test we used Student's $t$-test, or MannWhitney $U$-test. Categorical variables were compared by $\chi^{2}$-test or Fisher's exact test. Survival was defined as time from diagnosis until death or last follow-up. Survival curves were plotted using Kaplan-Meier method and log-rank test was used for comparison. Multivariate analysis was performed by multivariate Cox model. All statistical analyses were performed using Graphpad Prism 6 (GraphPad Software, Inc., La Jolla, CA, USA), SPSS (version 19.0) software (IBM Corporation, Armonk, NY, USA) and Microsoft Office 2010 software for Windows (Microsoft Corporation, Redmond, WA, USA). Statistical significance was defined as $P$-value less than 0.05 . 

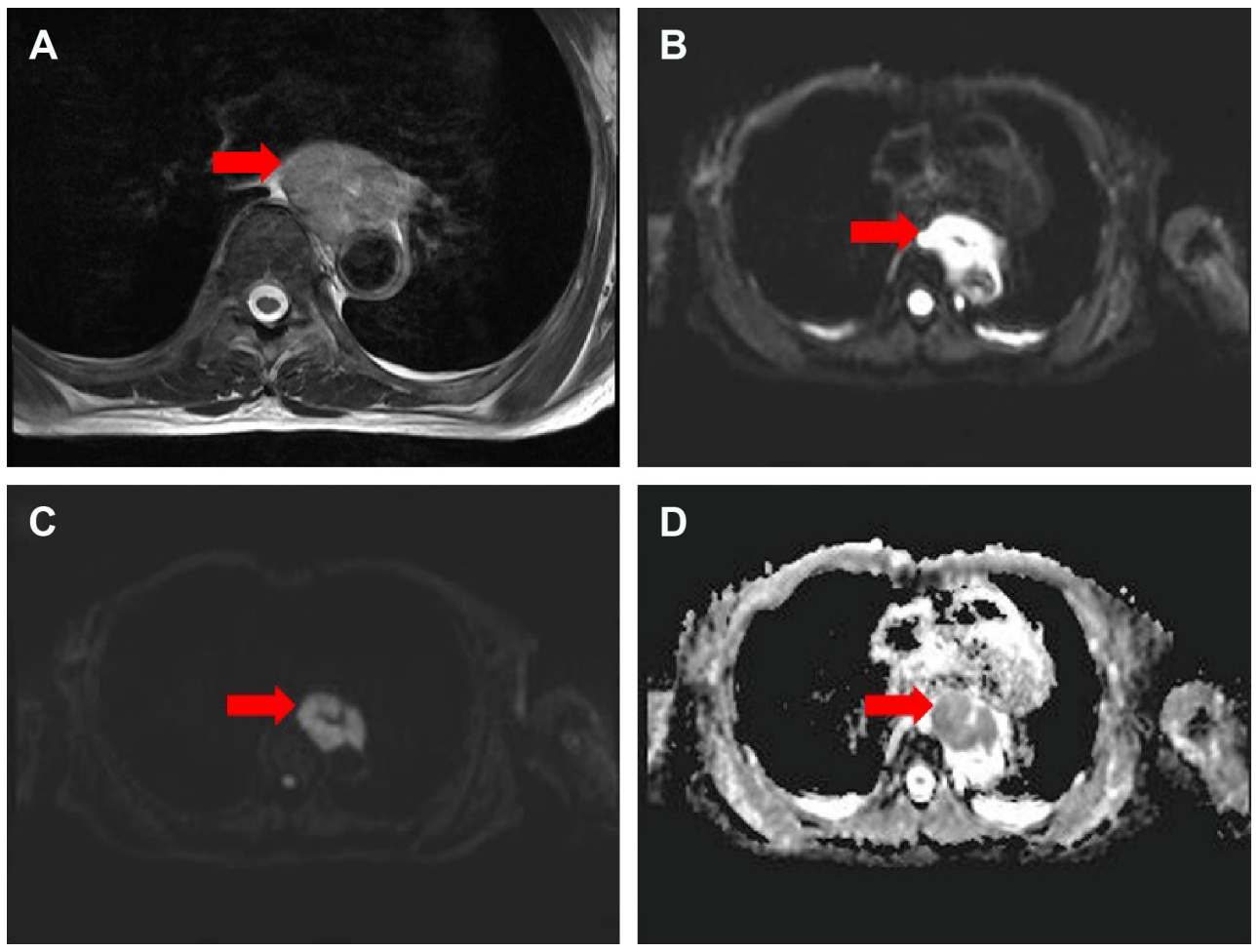

Figure I A 74-year-old female with a lesion involving the middle third of the esophagus.

Notes: The T2 image (A) showed an irregular wall thickening (arrow), native DWI images at b-values of 0 (B) and 800 sec/mm² (C) showed hyperintense signal and ADC map (D) showed hypointense signal. The average ADC value was $1.019 * 10^{-3} \mathrm{~mm}^{2} / \mathrm{s}$.

Abbreviations: ADC, apparent diffusion coefficient; DWI, diffusion-weighted imaging.

\section{Results}

The median ADC value of the esophageal cancer tissue was $1.256^{*} 10^{-3} \mathrm{~mm}^{2} / \mathrm{sec}$ (range: $0.657-2.354 * 10^{-3} \mathrm{~mm}^{2} / \mathrm{sec}$, interquartile range $\left.0.606 * 10^{-3} \mathrm{~mm}^{2} / \mathrm{sec}\right)$. The ADC value of cancer of the lower esophagus was significantly higher than that of cancer of the middle esophagus or cancer of the upper esophagus ( $P=0.0062$, Table 1$)$. No association was found between ADC value and other clinical variables (Table 1). The ADC of patients who have complete response (CR) to chemoradiation was not significantly different from that of patients who did not achieve CR (Table 1).

\section{Survival analysis}

While using univariate analysis, we found that presence of lymph node metastasis was significantly associated with a worse survival $(P=0.014$, hazard ratio [HR] 2.826). There was a trend that patients with $\mathrm{T} 4$ diseases had a worse prognosis than those with $\mathrm{T} 1-3$ diseases $(P=0.081)$, although this difference was not statistically significant. The median survival of the patients who achieved CR was significantly higher than that of patients who did not $(P<0.0001$, $\mathrm{HR}=45.7)$. The survival of cancer of the middle esophagus
Table I The ADC values and clinical variables in 73 patients with ESCC

\begin{tabular}{|c|c|c|}
\hline Variable (n) & $\begin{array}{l}\text { ADC value }\left(10^{-3} \mathrm{~mm}^{2} / \mathrm{s}\right) \\
\text { (median, interquartile } \\
\text { range) }\end{array}$ & $P$-value \\
\hline Sex & & 0.6743 \\
\hline Male (54) & $1.274,0.602$ & \\
\hline Female (19) & $1.233,0.657$ & \\
\hline Age & & 0.7821 \\
\hline$\leq 60(24)$ & $1.282,0.592$ & \\
\hline$>60(49)$ & $1.252,0.649$ & \\
\hline Tumor depth & & 0.054 \\
\hline TI-T3 (35) & I.37I, 0.704 & \\
\hline T4 (38) & I. $175,0.409$ & \\
\hline $\mathrm{N}$ factor & & 0.6601 \\
\hline No (44) & $1.232,0.606$ & \\
\hline NI (29) & $1.308,0.576$ & \\
\hline CRT effect & & 0.2199 \\
\hline CR (58) & $1.269,0.624$ & \\
\hline PR, PD or SD (I5) & $1.087,0.456$ & \\
\hline Location & & 0.0062 \\
\hline Upper (25) & $1.186,0.631$ & \\
\hline Middle (27) & I. $173,0.332$ & \\
\hline Lower (2I) & $1.555,0.708$ & \\
\hline
\end{tabular}

Abbreviations: $A D C$, apparent diffusion coefficient; ESCC, esophageal squamous cell carcinoma; $\mathrm{CRT}$, chemoradiation therapy; $\mathrm{N}$, lymph node; $\mathrm{CR}$, complete response; $\mathrm{PR}$, partial response; SD, stable disease; PD, progressed disease. 


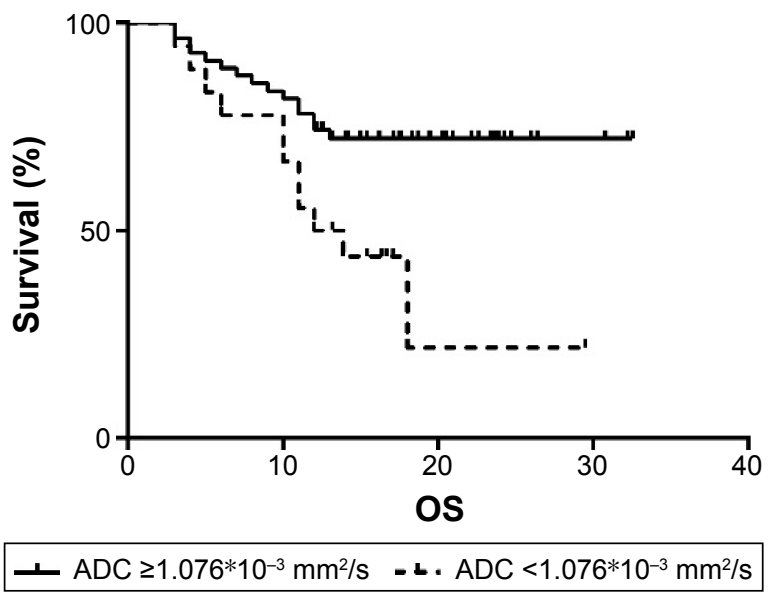

Figure 2 Low ADC value predicted worse survival in patients with ESCC. Notes: Patients with ADC $<1.076^{*} 10^{-3} \mathrm{~mm}^{2} / \mathrm{s}$ had a significantly worse overall survival than those with $A D C \geq 1.076 * 10^{-3} \mathrm{~mm}^{2} / \mathrm{s}$.

Abbreviations: ADC, apparent diffusion coefficient; OS, overall survival; ESCC, esophageal squamous cell carcinoma.

was worse than that of those from other sites (13.9 months vs undefined), although the difference is not statistically significant $(P=0.0537)$. We determined if ADC was prognostic in our cohort. According to the previous study, we used $1.1 * 10^{-3} \mathrm{~mm}^{2} / \mathrm{sec}$ as the cutoff of ADC; however, the survival between patients with $\mathrm{ADC}<1.1 * 10^{-3} \mathrm{~mm}^{2} / \mathrm{sec}$ and patients with $\mathrm{ADC} \geq 1.1 * 10^{-3} \mathrm{~mm}^{2} / \mathrm{sec}$ was not significantly different $(P=0.1517)$. We use X-tile to find a cutoff of ADC to best predict the prognosis of these patients. ${ }^{4}$ Using 1.076 as the cutoff value of $\mathrm{ADC}$, we divided our patients into two groups, we found that the overall survival of patients with $\mathrm{ADC}<1.076^{*} 10^{-3} \mathrm{~mm}^{2} / \mathrm{sec}$ was significantly worse than patients with $\mathrm{ADC} \geq 1.076^{*} 10^{-3} \mathrm{~mm}^{2} / \mathrm{sec}$ (12.9 months vs undefined, $P=0.0108$, Figure 2). We then determined whether ADC was an independent factor that was predictive of survival. We included age, sex, tumor depth, lymph node metastasis, and ADC into multivariate Cox analysis. In multivariate analysis, ADC $<1.076^{*} 10^{-3} \mathrm{~mm}^{2} / \mathrm{sec}$ was an independent predictor of worse survival in our cohort (Table 2, $P=0.014$; HR 2.715 [1.226-6.014]).

Table 2 Factors predicting survival in 73 patients

\begin{tabular}{lll}
\hline Variables & \multicolumn{2}{l}{ Multivariate analysis } \\
\cline { 2 - 3 } & HR $(95 \% \mathrm{CI})$ & P-value \\
\hline Sex & $2.167(0.723-6.492)$ & 0.167 \\
Age & $1.546(0.659-3.630)$ & 0.317 \\
Tumor size & $2.292(0.997-5.27 I)$ & $0.05 I$ \\
N factor & $2.744(1.224-6.149)$ & 0.014 \\
ADC & $2.715(1.226-6.014)$ & 0.014 \\
\hline
\end{tabular}

Abbreviations: $\mathrm{N}$, lymph node; $\mathrm{ADC}$, apparent diffusion coefficient; $\mathrm{HR}$, hazard ratio; $\mathrm{Cl}$, confidence interval.

\section{Discussion}

The diagnostic performance of DWI was first investigated in ESCC. ${ }^{5}$ The study by Sakurada et al found that DWI only has a limited role in detecting esophageal cancer or lymph node metastasis. ${ }^{5}$ Several studies have established the prognostic value of ADC in ESCC. The study by Aoyaqi et al first demonstrated that the ADC value was useful in predicting treatment response as well as survival for patients with ESCC receiving chemoradiotherapy. ${ }^{2}$ Furthermore, the ADC change after treatment was also proven to be a useful marker for evaluating therapeutic responses in several types of cancer. ${ }^{6}$ The study by Priola et al demonstrated that, in patients with thymoma, patients with higher ADC have a significantly higher diseasefree survival than patients with lower ADC. ${ }^{7}$ In a cohort of 41 patients with head and neck squamous cell carcinoma treated with radiotherapy, ADC calculated with different b-values significantly correlated with disease-free survival or overall survival. ${ }^{8}$ Furthermore, in patients with resectable gastric cancer, lower ADC was significantly associated with a negative prognosis. ${ }^{9}$

In our study, the range of ADC was 0.657-2.354. The range of $\mathrm{ADC}$ values of this cohort was larger than that of the study by Aoyagi et al (range: $0.36-1.86){ }^{2}$ The range of ADC values of our cohort was also larger than that of the study by Kwee et al, although the exact maximum and minimum value was not documented. ${ }^{10}$ The range was similar to that of the study by Giganti et al. ${ }^{3}$ The variations of ADC values in these studies could be due to different instruments, different software or different b-values used. We found that low ADC value predicted poor survival in patients with ESCC. However, we have not confirmed the association between $\mathrm{ADC}$ value and therapeutic response, probably due to the high CR rate in our cohort. We also found that the ADC value of cancer of the lower esophagus was significantly higher than that of cancer of the middle esophagus or cancer of the upper esophagus, a novel finding that was not previously reported.

The ADC value indicates the diffusion of water in tissues. Several factors, including hypoxia, inflammation, cell density and cell membrane integrity, can affect the diffusion of water in tissues. ${ }^{11}$ Previous studies indicated that ADC values inversely correlate with cell density in several malignancies. ${ }^{12}$ As a result, low $\mathrm{ADC}$ may reflect the aggressiveness of the tumor. ${ }^{3}$ And in ESCC, the ADC values decreased with stromal collagen growth; also, a negative correlation was noted between the ADC of tumors and the level of VEGF in tumor tissues, suggesting that low ADC was associated with increased angiogenesis. ${ }^{13}$ In patients with esophageal 
carcinoma, elevated angiogenic-factor expression was found to be associated with inferior treatment response and adverse outcome. ${ }^{14}$ Combination of earlier studies may partly explain the observation that low ADC value predicted worse outcome in patients with ESCC. ${ }^{13,14}$

However, low ADC value does not always predict worse outcome in other types of cancer. For instance, in patients with nasopharyngeal carcinoma, high pretreatment ADC predicted poor local relapse-free survival and disease-free survival. ${ }^{15}$ And Zhang et al found that nasopharyngeal carcinoma patients with low pretreatment ADC responded better to neoadjuvant chemotherapy. ${ }^{16}$ These might be due to different pathophysiologies in different types of cancer. As a result, the prognostic value of ADC should be interpreted depending on different types of cancer.

However, our study has several limitations. First, the cohort of this study is relatively small, and it may have caused nonsignificance of some results. Second, we used perfusion-sensitive ADC values, because we included b-values of $0 \mathrm{~mm} / \mathrm{sec}^{2}$ in ADC maps without suppression of tissue perfusion. ${ }^{17-20}$ Thus, the ADC values in our study may be overestimated. Third, although we excluded macroscopic vessels from ROI positioning, microscopic vessels and capillaries may have been included in ROI freehand area of measurement. ${ }^{17-20}$

\section{Conclusion}

In conclusion, our study confirmed $\mathrm{ADC}$ as a prognostic factor in ESCC patients who received chemoradiation. Our results suggest MRI examination using DWI measurements should be performed before the treatment of ESCC patients.

\section{Author contributions}

All authors contributed to the design of the study, data analysis, drafting and revising the manuscript, and gave final approval for the version to be published, and agree to be responsible for all aspects of the work.

\section{Disclosure}

The authors report no conflicts of interest in this work.

\section{References}

1. Cohen DJ, Leichman L. Controversies in the treatment of local and locally advanced gastric and esophageal cancers. J Clin Oncol. 2015; 33(16): 1754-1759.

2. Aoyagi T, Shuto K, Okazumi S, Shimada H, Kazama T, Matsubara H. Apparent diffusion coefficient values measured by diffusion-weighted imaging predict chemoradiotherapeutic effect for advanced esophageal cancer. Dig Surg. 2011;28(4):252-257.
3. Giganti F, Salerno A, Ambrosi A, et al. Prognostic utility of diffusionweighted MRI in oesophageal cancer: is apparent diffusion coefficient a potential marker of tumour aggressiveness? Radiol Med. 2016;121(3): 173-180.

4. Camp RL, Dolled-Filhart M, Rimm DL. X-tile: a new bio-informatics tool for biomarker assessment and outcome-based cut-point optimization. Clin Cancer Res. 2004;10(21):7252-7259.

5. Sakurada A, Takahara T, Kwee TC, et al. Diagnostic performance of diffusion-weighted magnetic resonance imaging in esophageal cancer. Eur Radiol. 2009;19(6):1461-1469.

6. Sun YS, Cui Y, Tang L, et al. Early evaluation of cancer response by a new functional biomarker: apparent diffusion coefficient. AJR Am J Roentgenol. 2011;197(1):W23-W29.

7. Priola AM, Priola SM, Giraudo MT, et al. Diffusion-weighted magnetic resonance imaging of thymoma: ability of the Apparent Diffusion Coefficient in predicting the World Health Organization (WHO) classification and the Masaoka-Koga staging system and its prognostic significance on disease-free survival. Eur Radiol. 2016;26(7):2126-2138.

8. Hatakenaka M, Nakamura K, Yabuuchi H, et al. Apparent diffusion coefficient is a prognostic factor of head and neck squamous cell carcinoma treated with radiotherapy. Jpn J Radiol. 2014;32(2):80-89.

9. Giganti F, Orsenigo E, Esposito A, et al. Prognostic role of diffusionweighted MR imaging for resectable gastric cancer. Radiology. 2015; 276(2):444-452.

10. Kwee RM, Dik AK, Sosef MN, et al. Interobserver reproducibility of diffusion-weighted MRI in monitoring tumor response to neoadjuvant therapy in esophageal cancer. PLoS One. 2014;9(4):e92211.

11. Hatakenaka M, Nakamura K, Yabuuchi H, et al. Pretreatment apparent diffusion coefficient of the primary lesion correlates with local failure in head-and-neck cancer treated with chemoradiotherapy or radiotherapy. Int J Radiat Oncol Biol Phys. 2011;81(2):339-345.

12. Squillaci E, Manenti G, Cova M, et al. Correlation of diffusion-weighted MR imaging with cellularity of renal tumours. Anticancer Res. 2004; 24(6):4175-4179.

13. Aoyagi T, Shuto K, Okazumi S, et al. Apparent diffusion coefficient correlation with oesophageal tumour stroma and angiogenesis. Eur Radiol. 2012;22(6):1172-1177.

14. Oshima Y, Yajima S, Yamazaki K, Matsushita K, Tagawa M, Shimada H. Angiogenesis-related factors are molecular targets for diagnosis and treatment of patients with esophageal carcinoma. Ann Thorac Cardiovasc Surg. 2010;16(6):389-393.

15. Zhang Y, Liu X, Zhang Y, et al. Prognostic value of the primary lesion apparent diffusion coefficient (ADC) in nasopharyngeal carcinoma: a retrospective study of 541 cases. Sci Rep. 2015;5:12242.

16. Zhang GY, Wang YJ, Liu JP, et al. Pretreatment diffusion-weighted MRI can predict the response to neoadjuvant chemotherapy in patients with nasopharyngeal carcinoma. Biomed Res Int. 2015;2015: 307943.

17. Priola AM, Priola SM, Gned D, et al. Diffusion-weighted quantitative MRI to diagnose benign conditions from malignancies of the anterior mediastinum: improvement of diagnostic accuracy by comparing perfusion-free to perfusion-sensitive measurements of the apparent diffusion coefficient. J Magn Reson Imaging. Epub 2016 Feb 19.

18. Koh DM, Collins DJ. Diffusion-weighted MRI in the body: applications and challenges in oncology. AJR Am J Roentgenol. 2007;188(6): 1622-1635.

19. Padhani AR, Liu G, Koh DM, et al. Diffusion-weighted magnetic resonance imaging as a cancer biomarker: consensus and recommendations. Neoplasia. 2009;11(2):102-125.

20. Priola AM, Gned D, Veltri A, Priola SM. Chemical shift and diffusionweighted magnetic resonance imaging of the anterior mediastinum in oncology: current clinical applications in qualitative and quantitative assessment. Crit Rev Oncol Hematol. 2016;98:335-357. 


\section{Publish your work in this journal}

OncoTargets and Therapy is an international, peer-reviewed, open access journal focusing on the pathological basis of all cancers, potential targets for therapy and treatment protocols employed to improve the management of cancer patients. The journal also focuses on the impact of management programs and new therapeutic agents and protocols on

patient perspectives such as quality of life, adherence and satisfaction. The manuscript management system is completely online and includes a very quick and fair peer-review system, which is all easy to use. Visit http://www.dovepress.com/testimonials.php to read real quotes from published authors.

Submit your manuscript here: http://www.dovepress.com/oncotargets-and-therapy-journal 